\title{
TIDAL CIRCULARIZATION AMONG THE CLOSE BINARIES IN THE HALO
}

\author{
Guillermo Torres \\ Harvard-Smithsonian Center for Astrophysics and \\ Córdoba Observatory, National University of Córdoba \\ Laprida 854, 5000 Córdoba, Argentina \\ David W. Latham \\ Harvard-Smithsonian Center for Astrophysics \\ 60 Garden Street, Cambridge, Massachusetts 02138, U.S.A.
}

\section{Tsevi Mazeh}

School of Physics and Astronomy, Raymond and Beverly Sackler Faculty of Exact Science Tel Aviv University, Tel Aviv 69978, Israel

\author{
Bruce W. Carney \\ Department of Physics and Astronomy, University of North Carolina \\ Chapel Hill, North Carolina 27599-3255, U.S.A.
}

Robert P. Stefanik and Robert J. Davis

Harvard-Smithsonian Center for Astrophysics

60 Garden Street, Cambridge, Massachusetts 02138, U.S.A.

John B. Laird

Department of Physics and Astronomy, Bowling Green State University

Bowling Green, Ohio 43403, U.S.A.

\begin{abstract}
For a sample of halo binaries, we find that the transition between circular and eccentric orbits occurs at a period of at least 18.7 days. This is consistent with the Goldman \& Mazeh theory of
\end{abstract} tidal circularization on the main sequence.

\section{INTRODUCTION}

It is well known that short-period binaries usually have circular orbits, while long-period binaries have orbits with a wide distribution of eccentricities. This effect was noticed already by Campbell (1910), and was shown clearly by Koch and Hrivnak (1981) for a sample of late-type spectroscopic binaries taken from the catalog of Batten et al. (1978). This phenomenon has usually been attributed to tidal mechanisms, acting to circularize the orbits of short-period binaries (e.g. Zahn 1977).

Mayor and Mermilliod (1984) were the first to study the distribution of orbital eccentricity in a coeval sample of binaries. They found that in the Hyades, Praesepe, and Coma, three open clusters with 
the same age, all the orbits for main-sequence binaries with periods shorter than 5.6 days were circular. Mathieu and Mazeh (1988) pointed out that for an older sample, where the tidal mechanisms have worked for a longer time, the transition period should be longer. They proposed that the transition period might be used as a kind of clock for estimating the relative ages of various coeval populations of binaries.

On the theoretical side, there has been considerable discussion of how the circularization timescale should depend on orbital period. For example, Zahn (1977) and Mathieu and Mazeh (1988) argue that the transition period should vary as $P^{16 / 3}$, while Tassoul (1988) suggested a milder $P^{49 / 12}$ dependence. More recently, Goldman and Mazeh (1991) have proposed that the transition period should vary in a still milder way, as $P^{10 / 3}$. These theories only apply to stars with convective envelopes and not to stars that are so massive that their envelopes are purely radiative.

When stars are in the process of formation by collapse along their Hayashi tracks, they are much larger and have extended convective zones. Zahn and Bouchet (1988) argued that tidal circularization would be much more important during this pre-main-sequence (PMS) history of a binary than after it reached the main sequence. They predicted that the transition period should be between 7.2 and 8.5 days, independent of the amount of time the sample has spent on the main sequence.

On the observational side, transition periods have been determined for just a few coeval samples, and not very precisely, despite considerable effort to derive more orbits for binaries in the critical range of periods (e.g. see Mazeh et al. 1990). In addition, Duquennoy and Mayor (1992) have recently emphasized that any evolution of the orbital eccentricity must also be accompanied by evolution of the period. In general the period of a binary will grow shorter as the orbit becomes more circular, and in a coeval sample there may be a range in transition period, over which there are both circular and eccentric orbits, instead of a single sharp transition from circular to eccentric orbits. Orbits with high initial eccentricity will take longer to circularize than orbits with the same initial period but less extreme initial eccentricity. Thus there can be eccentric orbits which are still in the process of being circularized but with shorter periods than a circularized orbit that started out with a less extreme eccentricity. Duquennoy and Mayor argue that, until it is possible to model the period evolution of a binary in detail, it is best to use the longest circular orbit as a lower limit for the transition period.

With the new definition proposed by Duquennoy and Mayor (1992), the transition period becomes at least 12.4 days for the old open cluster M67 (Latham et al. 1992). In this paper we determine the transition period for a very old sample of metal-poor halo binaries found in the Carney-Latham propermotion survey (Carney \& Latham 1987 et seq.). For these binaries we find that the transition period is at least $\mathbf{1 8 . 7}$ days.

\section{THE SAMPLE}

For ten years we have been monitoring the radial velocities of a sample of about $1450 \mathrm{~F}, \mathrm{G}$, and $\mathrm{K}$ dwarfs selected on the basis of their high proper motions, with a typical precision of better than $1 \mathrm{~km} \mathrm{~s}^{-1}$. So far we have derived spectroscopic orbits for more than 150 binaries and multiple systems (Latham et al. 1988; Latham et al. 1991), with semiamplitudes as low as $2 \mathrm{~km} \mathrm{~s}^{-1}$. The same spectra from which we determine the radial velocities are also used to extract spectroscopic metallicities for most of our stars (Carney $e t$ al. 1987), most of which are metal poor. Figure 1 shows the metallicity distribution for the entire propermotion sample, where the peak near $[\mathrm{m} / \mathrm{H}]=-1.5$ corresponds to the halo (Laird et al. 1988), and the more-pronounced peak near $[\mathrm{m} / \mathrm{H}]=-0.5$ is mostly due to the thick disk (Carney et al. 1989).

Because our proper-motion selection has introduced a strong kinematic bias, we have chosen to use metallicity to pick out the stars in the halo. If we restrict ourselves to stars more metal-poor than $[\mathrm{m} / \mathrm{H}]=-1.5$, then we can avoid most of the overlap with the metallicity distribution of the thick disk. There can be little doubt that the majority of the stars which are more metal-poor than $[\mathrm{m} / \mathrm{H}]=-1.5$ are among the oldest in our Galaxy. Of course, there may be a significant spread in the ages of our halo stars, and our sample of metal-poor binaries can not be strictly coeval. Hopefully the spread in age for our sample is small compared to the age of the Galaxy. 


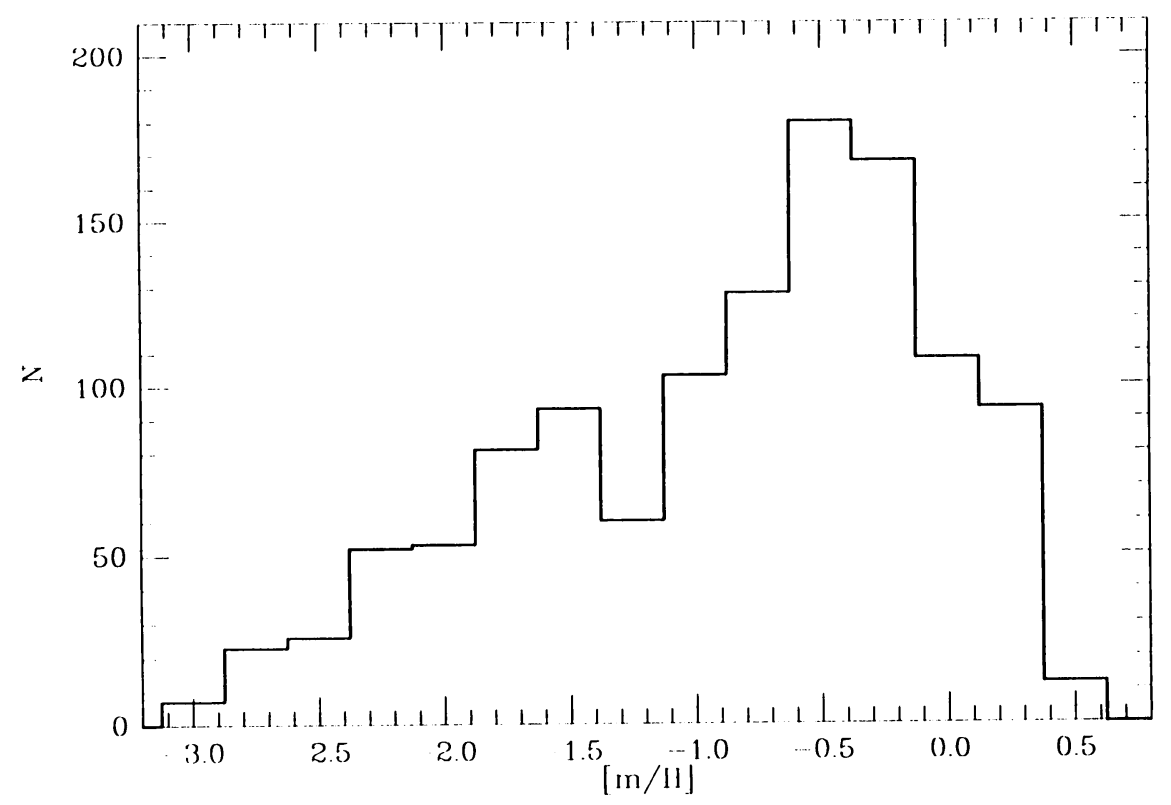

Fig. 1. Histogram of the metallicities for all the stars in the proper-motion survey.

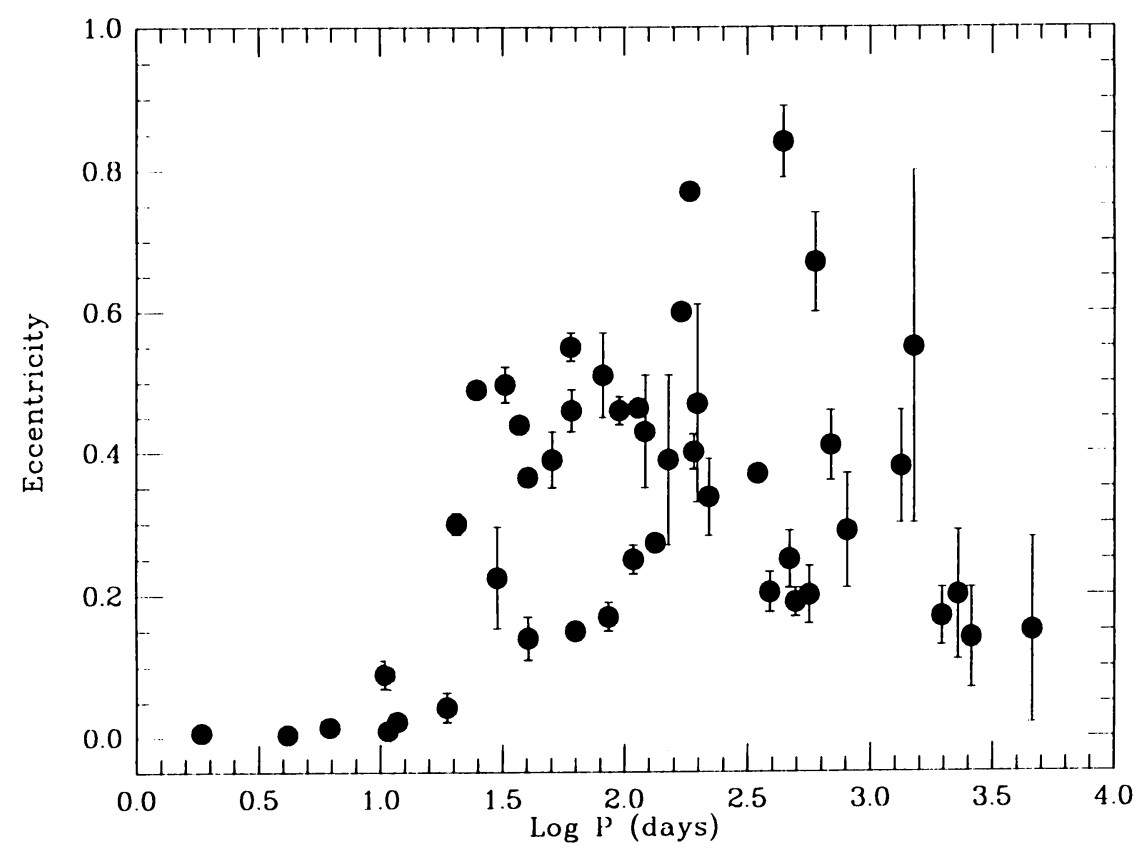

Fig. 2. Eccentricity versus $\log$ period diagram for the halo binaries with $[\mathrm{m} / \mathrm{H}]<-1.5$. 
Table 1. Orbital periods and eccentricities for our halo binaries

\begin{tabular}{|c|c|c|c|c|c|}
\hline Name & $\alpha$ & $(1950) \delta$ & Period & eccentricity & Comments \\
\hline G30-52 & $0: 09: 55$ & $+14: 17: 18$ & 1.84 & $0.007 \pm 0.008$ & \\
\hline G158-104 & $0: 35: 32$ & $-7: 21: 24$ & 4.16 & $0.004 \pm 0.008$ & \\
\hline G183-9 & 17:50:44 & $+15: 21: 42$ & 6.20 & $0.006 \pm 0.006$ & DLSB \\
\hline G176-46B & $11: 38: 37$ & $+39: 35: 54$ & 10.44 & $0.090 \pm 0.020$ & TRIPLE \\
\hline G66-59 & $15: 01: 24$ & $+10: 56: 12$ & 10.74 & $0.010 \pm 0.010$ & DLSB \\
\hline G176-27 & 11:19:03 & $+50: 54: 06$ & 11.73 & $0.023 \pm 0.008$ & DLSB \\
\hline G65-22 & 13:59:15 & +9:10:18 & 18.74 & $0.043 \pm 0.021$ & \\
\hline G88-10 & $7: 07: 20$ & $+24: 25: 42$ & 20.63 & $0.262 \pm 0.030$ & DLSB \\
\hline G122-43 & 11:41:40 & $+40: 49: 06$ & 24.85 & $0.490 \pm 0.010$ & \\
\hline G190-10 & $23: 05: 38$ & $+41: 35: 12$ & 30.15 & $0.225 \pm 0.071$ & \\
\hline G206-34 & $18: 33: 21$ & $+28: 39: 06$ & 32.39 & $0.497 \pm 0.025$ & \\
\hline G161-82 & $9: 48: 56$ & $-4: 24: 18$ & 37.13 & $0.440 \pm 0.010$ & DLSB \\
\hline
\end{tabular}

\section{ECCENTRICITY VERSUS PERIOD FOR OUR HALO BINARIES}

The eccentricity versus log period diagram for all our orbital solutions of binaries more metal-poor than $[\mathrm{m} / \mathrm{H}]=-1.5$ is shown in figure 2 . In table 1 we list the corresponding orbital periods and eccentricities for the systems with periods less than about $\mathbf{4 0}$ days. The last circular orbit has a period of 18.7 days. Unfortunately, we only have a couple of halo binaries with periods near 19 days, and the number or orbital solutions needs to be increased. Such work is underway.

The binary G176-46B is especially interesting, because it has a small but significant eccentricity, $e=0.09 \pm 0.02$, despite the fact that the period, $P=10.44$ days, is quite a bit shorter than the transition period. It has been shown (Mazeh \& Shaham 1979, Mazeh 1990) that a third star in a wide orbit around a binary can pump up the eccentricity to about 0.1 or so. G176-46 is indeed a triple system, and the orbital elements listed in table 1 correspond to the closer pair. The third star is actually the brightest in the system, and the correlation peak corresponding to its spectrum has remained steady over the 10 years that we have monitored G176-46. The secondary correlation peak has a variable velocity corresponding to the single-lined orbital solution listed in table 1.

The halo binaries with periods longer than 19 days display a wide distribution of eccentricities, with a mean of $0.37 \pm 0.03$. This is consistent with the mean for the non-circularized binaries in the Hyades and Praesepe (Burki \& Mayor 1986), in M67 (Mathieu et al. 1990, Latham et al 1992), and for the solartype dwarfs in the solar neighborhood (Duquennoy \& Mayor 1991). Because we expect the longer-period binaries to be pretty good at retaining their initial eccentricities, we conclude that the initial distribution of eccentricities is very much the same in the halo as it is in the disk, despite significant differences in chemical composition and kinematics between these two populations.

\section{DISCUSSION}

In our halo sample there are three binaries with circular orbits, but with periods longer than the limit of 8.5 days that Zahn \& Bouchet (1989) predict from tidal circularization during the PMS phase, when the stars are still collapsing along their Hayashi tracks. Is it possible that these three binaries just happened to form with circular orbits? We don't think this is likely, because there are no corresponding binaries with circular orbits for the many systems with periods longer than 19 days. Did these three binaries start out with massive primaries, which then evolved to giants with extended convective envelopes? This might have circularized the orbits much more efficiently than on the main sequence. No, the fact that two of 
these binaries are double-lined means that their companions are not white dwarfs.

Thus, at least two samples of old binaries have a transition from circular to eccentric orbits at a period longer than the 8.5 days predicted by Zahn \& Bouchet (1989); M67 with an age of $5 \mathrm{Gyr}$ and transition period of at least 12.4 days (Latham et al. 1992), and our sample of halo binaries with an age of roughly $15 \mathrm{Gyr}$ and a transition period of at least 18.7 days. Perhaps Zahn \& Bouchet (1989) and Mathieu and Mazeh (1988) are both right. Maybe tidal circularization during the PMS phase dominates the transition period for younger samples of binaries, but when the age of the sample grows old enough, circularization on the main sequence can take over.

Can we use the two oldest samples to test the competing theories of tidal circularization on the main sequence? The halo of the Galaxy is usually thought to be about 3 times older than M67, based on models of stellar evolution and a comparison with globular clusters. For the Goldman and Mazeh theory of tidal circularization, where the age depends on the $10 / 3$ power of the transition period, the corresponding factor is $(18.7 / 12.4)^{10 / 3}=3.9$. This is considerably better agreement than the other theories, which give much larger factors.

We thank Joe Caruso, Ed Horine, Dick McCrosky, Jim Peters, Skip Schwartz, and Joe Zajac for help with the observations, and Yuval Krimolowski for help with the orbital solutions.

\section{REFERENCES}

Batten, A. H., Fletcher, J. M., \& Mann, P. J. 1978, Publ. Dom. Astrophys. Obs., 15, 121

Burki, G., \& Mayor, M. 1986, in IAU Symp. No. 118, Instrumentation and Research Programmes for Small Telescopes, eds. J. B. Hearnshaw \& P. L. Cottrell (Dordrecht: Reidel), p. 385

Campbell, W. W. 1910, Lick Obs. Bull., 6, 17

Carney, B. W., \& Latham, D. W. 1987, AJ, 92, 116

Carney, B. W., Laird, J. B., Latham, D. W., \& Kurucz, R. L. 1987, AJ, 94, 1066

Carney, B. W., Latham, D. W., \& Laird, J. B. 1989, 97, 423

Duquennoy, A., \& Mayor, M. 1991, A\&A, 248, 485

Duquennoy, A., \& Mayor, M. 1992, in Binaries as Tracers of Stellar Formation, ed. M. Mayor (Geneva, Geneva Obs.), in press

Goldman, I., \& Mazeh, T. 1991, ApJ, 376, 260

Koch, R. H., \& Hrivnak, B. J. 1981, AJ, 86, 438

Laird, J. B., Rupen, M. P., Carney, B. W., \& Latham, D. W. 1988, AJ, 96,1908

Latham, D. W., Mathieu, R. D., Milone, A. A. E., \& Davis, R. J. 1992, this volume

Latham, D. W., Mazeh, T., Carney, B. W., McCrosky, R. E., Stefanik, R. P., \& Davis, R. J. 1988, AJ, 96, 567.

Latham, D. W., Mazeh, T., Stefanik, R. P., Davis, R. J., Krimolowski, Y., \& Laird, J. B. 1991, in preparation.

Mathieu, R. D., Latham, D. W., \& Griffin, R. F. 1990, AJ, 100, 1859

Mathieu, R. D., \& Mazeh, T. 1988, ApJ, 326, 256

Mayor, M., \& Mermilliod, J.-C. 1984, in IAU Symp. No. 105, Observational Tests of the Stellar Evolution Theory, eds. A. Maeder \& A. Renzini (Dordrecht: Reidel), p. 411

Mazeh, T. 1990, AJ, 99, 675

Mazeh, T., Latham, D. W., Mathieu, R. D., \& Carney, B. W. 1990, in NATO Advanced Study Institute on Active Close Binaries, ed. C. Ibagnolu (Dordrecht: Kluwer), p. 145

Mazeh, T., \& Shaham, J. 1979, A\&A, 77, 145

Tassoul, J. L. 1988, ApJ, 324, L71

Zahn, J.-P. 1977, A\&A, 57, 383

Zahn, J.-P., \& Bouchet, L. 1989, A\&A, 223, 112 